\title{
ODC-FREE SOLVENT IMPLEMENTATION ISSUES FOR VULCANIZED RUBBER AND BOND SYSTEMS
}

\author{
James R. Hodgson \\ ATK / Thiokol Propulsion \\ Brigham City, Utah
}

\begin{abstract}
Thiokol Propulsion has worked extensively to replace 1,1,1-trichloroethane (TCA) with ODC-free solvents for use in the manufacture of the Reusable Solid Rocket Motor (RSRM) for the Space Shuttle Program. As Thiokol has transitioned from sub-scale to full-scale testing and implementation of these new solvents, issues have been discovered which have required special attention. The original intent of Thiokol's solvent replacement strategy was to replace TCA with a single drop-in solvent for all equivalent applications. We have learned that a single candidate does not exist for replacing TCA. Solvent incompatibility with process materials has caused us to seek for niche solvents and/or processing changes that provide an ODC-free solution for special applications.
\end{abstract}

This paper addresses some of the solvent incompatibilities, which have lead to processes changes and possible niche solvent usage. These incompatibilities were discovered during full-scale testing of ODC-free solvents and relate to vulcanized rubber and bond systems in the RSRM. Specifically, the following items are presented:

Cure effects of $d$-limonene based solvents on Silica Filled Ethylene Propylene Diene Monomer (SF-EPDM) rubber. During fullscale test operations, Thiokol discovered that d-limonene (terpene) based solvents inhibit the cure of EPDM rubber. Subsequent testing showed the same issue with Nitrile Butadiene Rubber (NBR). Also discussed are efforts to minimize uncured rubber exposure to solvents.

Copyright () 2001 ATK Thiokol Propulsion Corp.

Published by The American Institute of Aeronautics and Astronautics Inc. with permission.
Cured bond system sensitivity to ODCfree solvents. During full scale testing it was discovered that a natural rubber to steel vulcanized bond could degrade after prolonged exposure to ODC-free solvents. Follow on testing showed that low vapor pressure and residence time seemed to be most likely cause for failure.

\section{Introduction}

As with many development programs, Thiokol has employed sub-scale laboratory testing prior to fullscale testing in determining the best replacement solvent for 1,1,1-trichloroethane (TCA). Sub-scale testing was designed to simulate production cleaning and bonding on all of our substrates as well as characterize compatibility of the solvents with process materials. Common contaminates were cleaned from different substrates and bonded. These specimens were then tested for tensile adhesion strength as well as peel strength. Processes materials were also tested in the laboratory for compatibility by exposing the material to the solvent and testing for extractables, swelling and absorption. In the area of vulcanized rubber and bond systems, this testing was nearly complete. A total of eighty-one different candidate solvents were screened. After completing the majority of the sub-scale testing a preliminary down selection was made to RE-ENTRY@ Plus$4^{\mathrm{TM}}$.

After making the down selection to Plus-4 we were ready to begin testing the solvent on full-scale hardware. However, prior to building a full-scale static test rocket motor a series of interim full-scale process simulation articles were built. The purpose of process simulation articles is to understand how material changes may affect fabrication processes as well as gain experience 
using new materials before committing the resources for a complete rocket motor. These articles are processed through the standard manufacturing cycle up to the point just prior to bading propellant.

As often happens when using new processes or materials in full-scale operations, issues are uncovered that were not discovered during subscale testing. This was the case with the use of Plus -4 in the area of vulcanized rubber and bond systems. Incompatibilities between Plus-4 and uncured Silica Filled Ethylene Propylene Diene Monomer (SF-EPDM) were discovered during our scale-up testing. This was not discovered in subscale testing due to a difference in cure methods utilized. Autoclaves and vacuum bagging are used predominately throughout RSRM manufacture and as such were used exclusively in sub-scale testing. When simulating an obscure press cure operation during full-scale testing the incompatibility between Plus-4 and SF-EPDM was discovered. Another incompatibility discovered during full-scale testing was between natural rubber and steel bonds. Voids open to the outer surface of a full-scale test specimen allowed solvent to migrate into the void. Upon examination we discovered that entrapped Plus-4 can degrade the cured natural rubber to steel bond system. These issues discovered in full-scale testing have driven us to modify our processes to accommodate the change in solvent from TCA to Plus-4. In some cases, such as the natural rubber to steel bond system incompatibility, Plus- 4 simply cannot be used. Niche solvents are being investigated for these operations.

\section{Solvent Incompatibilities Discovered During Full-Scale Testing}

While building several full-scale process simulation articles two primary issues were discovered with the use of Plus-4 on rubber bonding. First, it was discovered that production bond simulators made with Plus-4 cleaned SFEPDM rubber had lower tensile strength capability than control specimens prepared with TCA. Secondly, cured natural rubber to steel bond systems degrade after prolonged exposure to Plus-4.
Press Cured Silica Filled EPDM to Steel

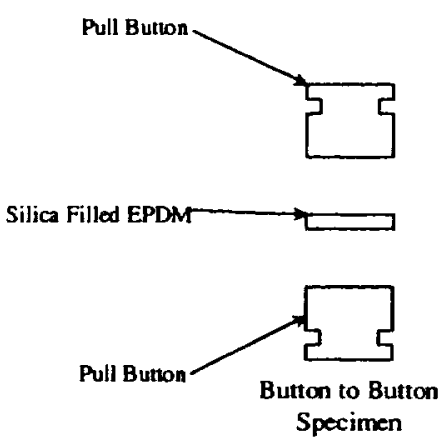

Figure 1 Press Cured SF-EPDM Test Specimens

\section{SF-EPDM cure interference by $d$ -}

\section{limonene}

The predominate cure method utilized at Thiokol to vulcanize rubber, is to vacuum bag the part and cure it under pressure in an autoclave. Because this is the primary method used in production for vulcanization it was used exclusively through out sub-scale testing. One of the full-scale test articles buitt during our scale-up testing uses a press cure as part of the normal production process. To better simulate the normal production flow used to fabricate this part a button-to-button test specimen was developed that utilized a press cure. The specimens were built by cleaning small discs of SF-EPDM with the candidate solvent, Plus-4, and sandwiching them between two tensile buttons (Figure 1). A control set was also prepared by cleaning the discs with TCA. The specimens were then press cured.

After the specimens were fabricated they were sent to the mechanical test laboratory for tensile testing on an Instron machine. The specimens were conditioned at ambient temperature, pressure and humidity prior to testing to failure. All of the specimens failed in the rubber and not at the bond interface. However, the data indicated as much as a $70 \%$ reduction of tensile strength capabilities for those specimens prepared with Plus-4 (Figure 2). Because a concurrent test effort was underway to replace the SF-EPDM used for the production part, several different candidate formulations of SF-EPDM together with the current baseline material were used for this test. There was substantial variation between the different formulations, though when directly compared to 


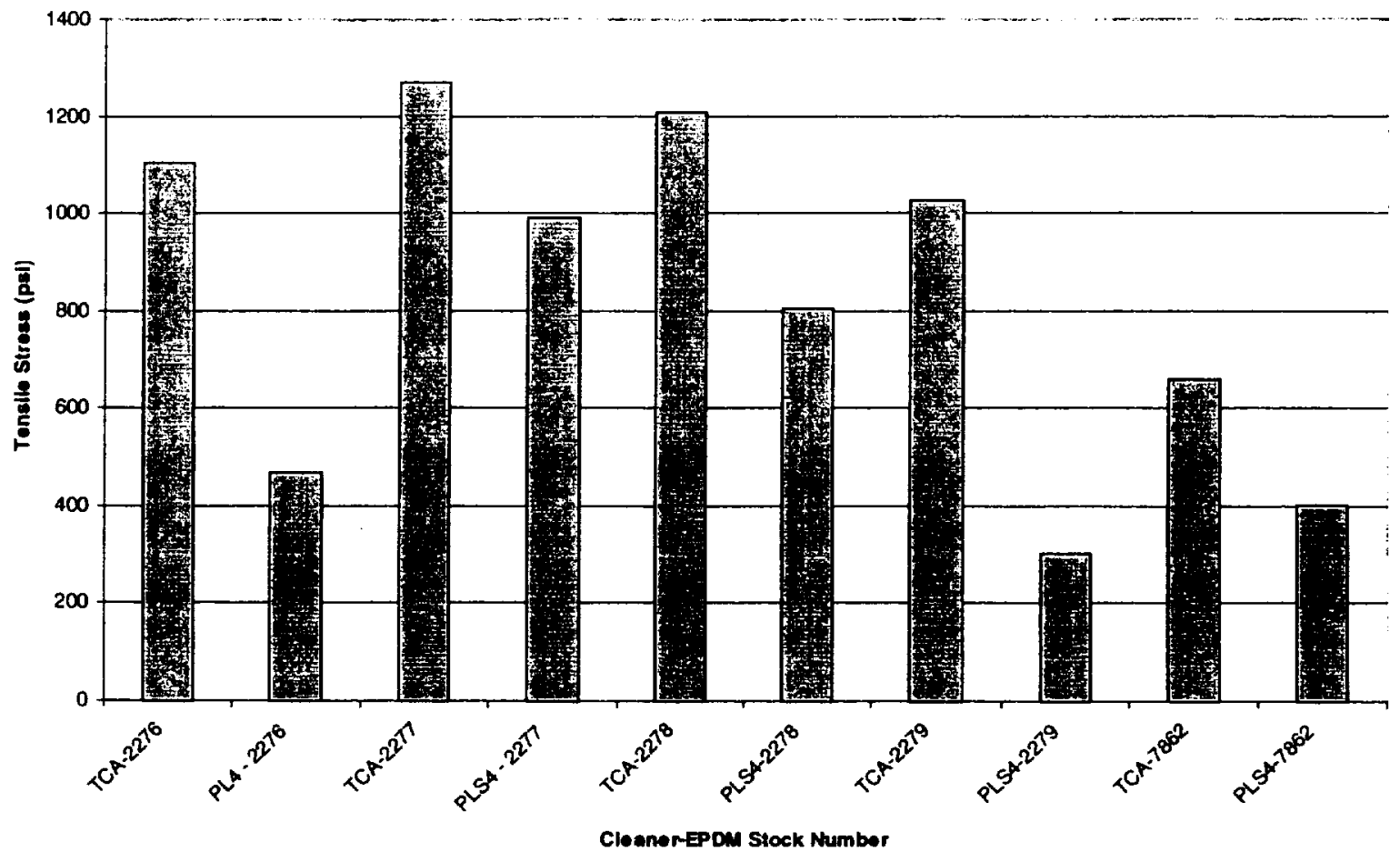

Figure 2 Press Cured Tensile Strength, SF-EPDM

the TCA prepared specimens, all of the Plus- 4 prepared specimens exhibited a reduction in tensile strength. The different materials are represented on Figure 2 by the four digit numbers associated with each set of data. Up to this point in our solvent replacement program sub-scale testing of SF-EPDM had shown no sensitivity to the solvent Plus-4. Uncured SF-EPDM had previously been cleaned with Plus-4 and subsequently bonded with acceptable results. The only discernable difference in testing methods was the use of the press cure process as opposed to the vacuum bagging and autoclave cure process. Because the autoclave cure processes uses a vacuum bag it was believed that, Plus- 4 being a volatile solvent, could be driven off and evacuated from the specimens during the cure process. By removing the Plus- 4 from the specimen in this manner it was not present during the actual cure reaction. Conversely, during a press cure there is no vacuum present to remove the solvent so it remains present during the cure reaction.

To further understand what may have caused the reduction in tensile strength, a Gas
Chromatograph/Mass Spectrometer (GC/MS) headspace was conducted on samples extracted from one of the test specimens. All of the constituents of Plus 4 were still found in the vulcanized rubber. This led us to believe that the Plus-4 solvent was responsible for the strength reduction. GC/MS was also used to show that the Plus- 4 found in the test specimens had a lower ratio of d-limonene than the base material. Dlimonene is the primary constituent of Plus-4. The reduction of d-limonene indicated that a reaction between it and the SF-EPDM had occurred.

In an effort to quantify the reduction in total cure caused by the d-limonene, additional testing was done using Gas Permeation Chromatography (GPC). Tetrahydrofuran (THF) was used to extract EPDM from specimens which had been wiped with Plus-4 and then cured. The idea behind this test was that once the SF-EPDM had cured, most of the rubber should be cross linked with the sulfur curative, tying up the EPDM and not allowing it to be extracted with THF. Two control specimens were used for this test. The first was a 


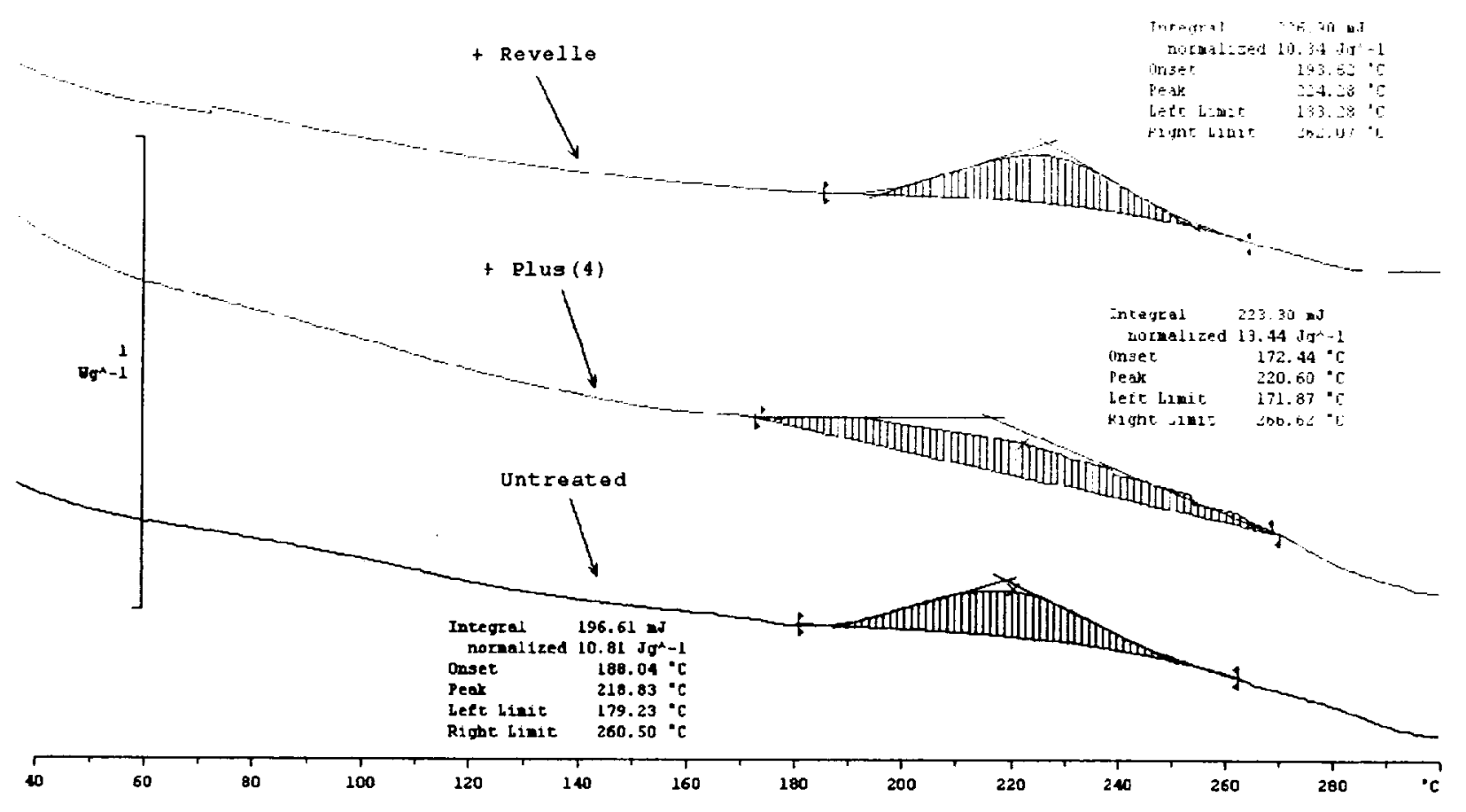

Figure 3 DSC for SF-EPDM cure reaction

zero point specimen of uncured SF-EPDM used to determine the max amount of EPDM that could be extracted with THF. The rest of the data was normalized to the zero point of the uncured specimen. The second, control was a cured specimen that had been wiped with TCA. The results of this testing showed that after cure the SF-EPDM specimens wiped with Plus-4 had significantly more EPDM available for extraction than did the TCA wiped specimens. This indicates that the Plus-4 prepared test specimens had not fully cured and in fact were much less cured than the TCA specimens. Normalizing the data to the uncured specimen as $0 \%$ cure and comparing the TCA and Plus-4 specimens, the TCA specimens were $95 \%$ cured while the Plus- 4 specimens were only $49 \%$ cured after press cure process. This testing indicated that some constituent of the Plus4 was inhibiting the SF-EPDM cure process.

A Differential Scanning Calorimeter (DSC) was used to evaluate the thermodynamics of the cure. As can be seen in Figure 3, the SF-EPDM that had not been exposed to any solvent exhibited an exotherm at approximately $193^{\circ} \mathrm{F}$. The SF-EPDM exposed to Reveille, a non-d-limonene based solvent used simply for comparison, showed no significant difference but the SF-EPDM exposed to
Plus-4 started to react at approximately $20^{\circ} \mathrm{F}$ lower temperature and continued over a much broader temperature range. Similar runs were made with each of the individual components of Plus 4. DLimonene was found to be the only component that indicate an interference in the cure reaction according to DSC testing.

In summary, all of our testing indicates that dlimonene, the primary constituent of Plus-4, reacts with the curative package of SF-EPDM rubber. The reaction reduces the amount of cure or cross linking of the rubber and this lack of cure is reflected in a low tensile strength.

After understanding the effects of d-limonene on SF-EPDM, similar press cured bond tests were conducted using Nitrile Butadiene Rubber (NBR). The results indicated that a similar reduction in strength occurs with NBR as occurs with SFEPDM.

\section{Mitigation efforts}

During typical uncured rubber operations, solvent cleaning of the rubber is not necessary. 
Therefore, the issue with incompatibility between Plus-4 and uncured rubber becomes more of a contamination control issue than a manufacturing difficulty. Accordingly, measures are being developed to mitigate concerns about using Plus-4 in areas where uncured rubber is present. The primary method of controlling the solvent will be the use of wipers pre-moistened with Plus-4. By using a pre-moistened wipe the amount of solvent used during an operation is controlled by the presaturation level in the cloth. Another advantage of using pre-moistened wipes is that out of place solvent is readily visible because it is in the form of a white wiper cloth. There is less chance of misplaced solvent going undetected with premoistened cloths. For manufacturing operations, which do not involve uncured rubber, squeeze bottles filled with solvent and dry wiper cloths will be available on an as needed basis. Using Premoistened wipes is the first option as a cleaning aid with squeeze bottles and dry wipes second.

In addition to using pre-moistened cloths, manufacturing planning will contain warnings about the incompatibility of Plus- 4 with uncured rubber. This coupled with educating the operations personnel responsible for the actual motor manufacture will help insure Plus-4 is not located near uncured rubber.

\section{Natural rubber to steel bond degradation}

After fabricating a process simulation article that incorporates a vulcanized natural rubber to steel bond line, the part was dissected because of a large number of voids discovered at the rubber to steel interface. These voids were inherent with the manufacturing process and not caused by the solvent being used. After dissecting this part internal voids that were opened to the outside had the distinct citrus smell associated with Plus-4. Because solvents are not allowed in the area during the build process, the only opportunity for solvent to enter the void was during post cure cleaning. During the post cure cleaning, copious amounts of solvent are used to remove cure residue. It was during this cleaning process that solvent migrated into voids which were open to the outer surface. Besides the citrus smell, the natural rubber that had been exposed to entrapped Plus-4 had discolored. However, after exposure to ambient air, the entrapped solvent evaporated and the discoloration faded until the rubber returned to its normal appearance.

Peel tests were performed on the rubber to steel interface in areas that had exhibited the discoloration and smell of Plus-4. This test was accomplished by cutting the rubber into strips approximately one-inch wide and pulling the strips from the steel at approximately a 90-degree angle. The results of this test showed that the rubber actually peeled near the edge of the void where the solvent had collected. Typically this test causes the rubber to tear at the edge of the void, it does not peel. The failure mode at the peel was thin film failure of the rubber at the adhesive interface. As this was an anomalous condition, further tests became necessary to understand the phenomenon.

A bench top laboratory test was developed which exposed natural rubber to steel bond line to entrapped solvents. After prolonged exposure to the solvent, peel tests were conducted on the specimens. The first test conducted was to duplicate in the laboratory what was witnessed on the full-scale hardware. This test was successful and further testing with different solvents was completed. Several ODC-free solvent candidates were tested and each of them exhibited the same short peel with thin film failure between the natural rubber and the adhesive. It was interesting to note that there was no single common component in any of the solvents. Similar tests conducted using TCA did not exhibit the same phenomenon. The TCA specimens all failed in the rubber at the edge of the void.

The characteristics of each of the tested solvents was examined and compared to TCA. The only common characteristic found was that each of the ODC-free candidate solvents had very low vapor pressures. The vapor pressures for these solvents ranged from approximately $1-2 \mathrm{mmHg}$ at ambient conditions. On the other hand TCA has a vapor pressure of approximately $100 \mathrm{mmHg}$ at ambient, which is nearly two orders of magnitude higher than the ODC-free candidates. It was hypothesized that the sensitivity of this bond line to the ODC-free solvent could be attributed to solvent residence time caused by low vapor pressure solvents. These solvents require much longer to dry than does TCA; therefore, they have a much greater chance to migrate into a void. 
Once trapped inside a void there is little chance for the solvent to evaporate away. The solvent will simply remain in the void degrading the bond between the natural rubber and the adhesive system.

While performing this testing, it was impossible, with the developed test configuration, to keep the TCA specimens wetted with the solvent during the exposure period. The question was raised whether or not TCA would exhibit the same phenomenon if the specimen remained wetted with the solvent. To answer this question a new test configuration was developed which allows assured solvent exposure and a better quantification of the results. This technique is still under development, however, preliminary results indicate that TCA does in fact cause the same failure as the ODC-free candidates if the solvent is in continuous contact with the rubber at the void interface. Even though TCA exhibited the same phenomenon, the fact that this type of failure has never been observed on other full-scale production parts provides strong evidence that high vapor pressure solvents such as TCA are the best choice for cleaning parts that incorporate a natural rubber to steel bond line.

Because the production part simulated by the process simulation article is intended for multiple reuses, any thing that can cause long-term degradation to the bond interfaces is unacceptable. Since this part is also susceptible to voids open to the outer surface, solvent entrapment is a likely occurrence. To date all of the ODC-free candidate solvents for replacing TCA investigated by Thiokol are low vapor pressure solvents. As such none of them can be considered for use in this operation.

\section{Mitigation efforts}

With no viable candidate solvents for use on parts incorporating natural rubber to steel bonds, identification of other potential solvents for manufacturing these parts has begun. A team has been commissioned to address this issue and prepare a proposed solution for testing on a fullscale static test motor.

This team is pursuing two primary efforts. The first is to change how vulcanized natural rubber parts are processed to reduce the amount of TCA needed to manufacture the parts. Changing processing to preclude the use of solvent is always the best ODC-free solution. The second effort being pursued by this team is identification of a niche solvent for use in cleaning vulcanized natural rubber parts. High vapor pressure solvents are being considered for this use. The team is currently evaluating several candidate solvents for this use. Compatibility testing and cleaning effectiveness are among the several characteristics presently being considered.

\section{Conclusions}

As Thiokol moves from the laboratory to full-scale implementation we have come to appreciate even more the indispensable nature of full-scale testing. Incompatibility between uncured SF-EPDM and natural rubber to steel bond systems are just two examples of issues discovered while conducting full-scale testing. Full-scale testing allowed us to catch these issues and resolve them before the changes were implemented into the flight program, at which point the cost of solving problems becomes infinitely more problematic and costly.

Additionally, we have learned that there is no single drop-in replacement for TCA. Certain operations, such as those involving natural rubber to steel bonds, will require specialty solvents. 